http://jmscr.igmpublication.org/home/ ISSN (e)-2347-176x ISSN (p) 2455-0450

crossref DOI: https://dx.doi.org/10.18535/jmscr/v7i10.90

Journal Of Medical Science And Clinical Research

\title{
A study on the epidemiological, clinical and laboratory profile of patients admitted with Dengue fever at a tertiary medical centre in South Kerala
}

Authors

\section{Padma Kumar Balasundaram, Sudharmma Rama Krishnan, Baraneedaran S}

\section{Background of the Study}

Globally 50 million dengue infections are reported annually ${ }^{[1]}$. In recent years Dengue has become one of the most important mosquito borne viral disease around the world. Female mosquitoes mainly of the species Aedes aegypti and, to a lesser extent, Aedes Albopictus transmits Dengue. Chikungunya, yellow fever and Zika infection are also transmitted by this mosquito. Due to local variations in risk influenced by rainfall, temperature and unplanned rapid urbanization dengue is widespread throughout the tropics ${ }^{[1]}$.

Dengue haemorrhagic fever was first recognized in the Philippines and Thailand in the 1950s during dengue epidemics. Today, severe dengue affects most Asian and Latin American countries and has become a leading cause of hospitalization and death ${ }^{[2]}$. The annual incidence is estimated to be 7.5 to 32.5 million in India $^{[3]}$. Dengue viruses (DV) belong to the family Flaviviridae. There are four serotypes of the virus DV-1, DV-2, DV-3, and DV $-4^{[2]}$. It is a positive-stranded encapsulated RNA virus. DV is composed of three structural protein genes. These structural protein genes encode the nucleocapsid or core $(\mathrm{C})$ protein, a membrane-associated $(\mathrm{M})$ protein, an enveloped (E) glycoprotein, and seven non-structural (NS) proteins. Infection with one dengue serotype provides lifelong homotypic immunity to that serotype. It also confers a very brief period of partial heterotypic immunity to other serotypes. But a person can eventually be infected by all 4 serotypes $^{[4]}$.

The disease spectrum may vary with asymptomatic illness to life threatening diseases like dengue haemorrhagic fever (DHF) and dengue shock syndrome (DSS).

The diagnosis can be made by IgM ELISA during recovery or by antigen-detection ELISA or RTPCR during the acute phase ${ }^{[14]}$. There are no specific management of dengue, other than supportive care.

\section{Indian Scenario}

Dengue fever (DF) probably was reported in India from Calcutta (now Kolkata), West Bengal in 1872. An epidemic of dengue hemorrhagic fever (DHF) was reported in Kolkata in July 1963. Around 0.1 million people were affected, mostly children with $40 \%$ case fatality rate in hospital admitted DHF cases. The first dengue haemorrhagic fever occurred in Calcutta during that epidemic. Since then several outbreaks have been reported in various states in India both the urban and rural areas. 


\section{Scenario in Kerala}

As early as 1979 dengue antibodies had been detected in human sera from Kozhikode, Kannur, Palakkad, Thrissur, Kottayam and Thiruvananthapuram districts. Sporadic occurrence of DF cases has been reported in Kerala since 1997 when 116 suspected cases with 4 deaths were reported from Kottayam. In 2001, a total of 877 suspected cases with one death were reported from four districts, viz. Kottayam, Idukki, Ernakulam and Thiruvananthapuram. Since then dengue fever has been regularly reported from Kerala. Dengue cases reported are higher during the months from June to October. The seasonality pattern has been maintained from the beginning of the epidemic throughout the years. In Kerala the highest number of cases are reported from Thiruvananthapuram. According to the data published by DHS the number of cases of dengue reported from Kerala are 2575 in 2014 and 4114 in 2015 out of which Kollam reported 73 and 245 cases respectively. Our study aims to study the clinical and laboratory profile of the patients admitted with dengue fever in Government Medical College Kollam.

\section{Objectives}

To study the clinical features and laboratory profile of patients with serologically confirmed dengue fever.

\section{Methods}

The study was a prospective observational study done in government medical college from September 2017 to September 2018. The study was conducted among all cases of confirmed dengue cases above the age of 12 years admitted in the department of medicine between September 2017 and September 2018. Approval was obtained from the intuitional ethical committee. Informed consent was obtained from all patients included in the study.

\section{Definition of Confirmed Case of Dengue Fever}

Clinical features of dengue with dengue NS $1 \mathrm{Ag}$ positive and/or IgM dengue positive using ELISA
Detailed history and careful clinical examination was performed on each patient. Laboratory investigations done were haemoglobin, total and differential leucocyte counts, platelet count, haematocrit, liver function tests, blood urea and serum creatinine, serum electrolytes and ECG. Serum lipase an amylase were done in indicated patients. Chest $x$ ray and ultra sound abdomen were done in selected cases as indicated. Blood counts and LFT were monitored periodically as and when required till resolution. Other differential diagnosis were excluded by appropriate tests.

Statistical analysis was made using SPSS software.

\section{Inclusion Criteria}

All patients above the age of 12 years with confirmed dengue fever admitted in the department of medicine during the study period were including in the study.

\section{Exclusion Criteria}

Those with concomitant malaria, leptospirosis, typhoid etc.

\section{Results}

The study included 175 patients above the age of 12 years admitted with dengue fever in the department of medicine from September 2017 to September 2018.

Majority of patients belonged to the 20 to 50 age group $(60.5 \%)$. among males the most common age group affected was 21-40 ages while among females the 31-50 age group was commonly infected.

There was only a slight male preponderance with $53.7 \%$ males and $46.3 \%$ females. It was notable that a significant $36 \%$ of the affected were homemakers suggesting that major source of breeding of Aedes mosquito was in the household itself. Manual labourers and students formed the next significant group affected. 


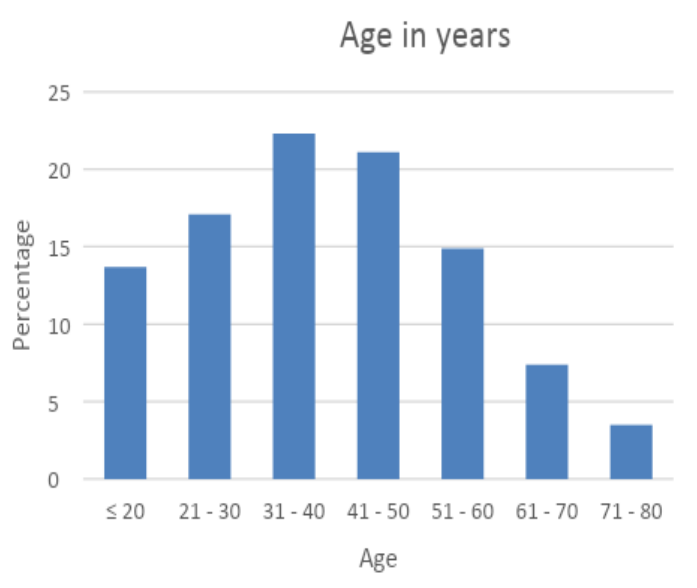

Figure 1 Age distribution of study population

Table 1 Sex distribution of the study population

\begin{tabular}{|l|c|c|}
\hline sex & Frequency & Percent \\
\hline Male & 94 & 53.7 \\
\hline Female & 81 & 46.3 \\
\hline Total & 175 & 100 \\
\hline
\end{tabular}

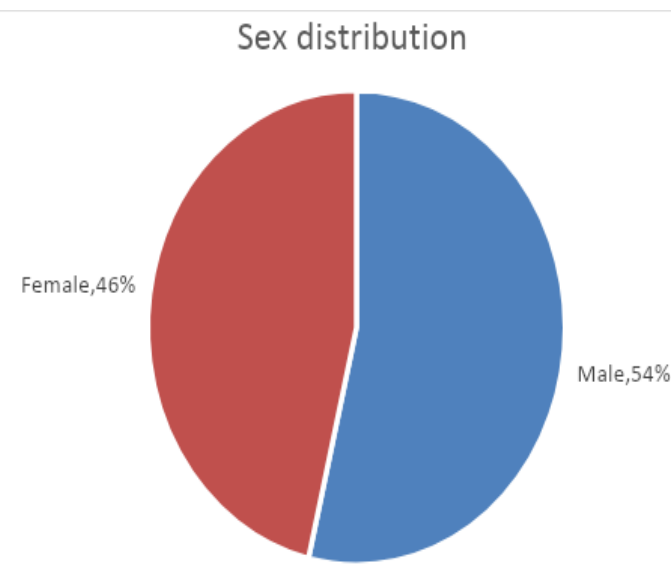

Fig 2 Sex distribution of the study population

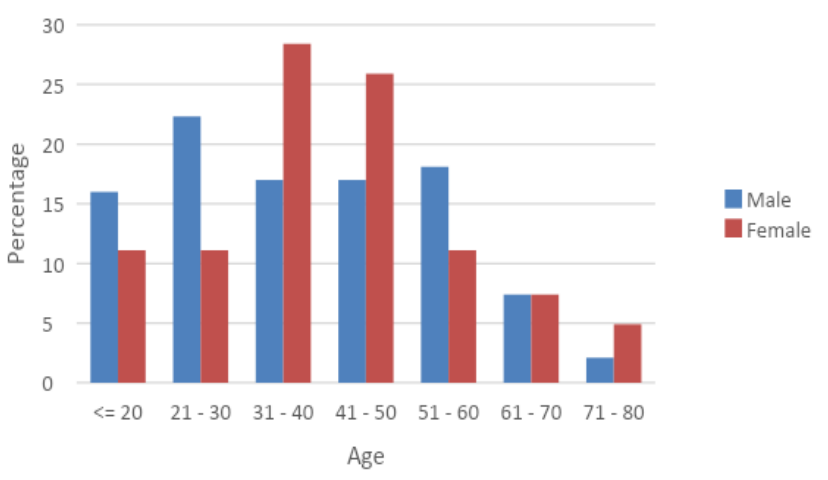

Fig 3 Age -sex distribution of the study population
Table 2 Occupation of the study population

\begin{tabular}{|l|c|c|}
\hline Occupation & Frequency & Percent \\
\hline Student & 31 & 17.7 \\
\hline Professional & 1 & 0.6 \\
\hline Manual Labourer & 42 & 24 \\
\hline Office work & 13 & 7.4 \\
\hline Housewife & 63 & 36 \\
\hline fisherman & 3 & 1.7 \\
\hline Factory work & 3 & 1.7 \\
\hline Others & 19 & 10.9 \\
\hline Total & 175 & 100 \\
\hline
\end{tabular}

Mosquito Control Practices and Waste Disposal Measures among Study Population

A meagre $4 \%$ of the study population conducted dry days once a week in their premises. The major method of waste disposal was burning of wastes in the premises of the house but a significant $41 \%$ of the study population admitted to open dumping of wastes. Only $11.4 \%$ of them had the facility of collection of wastes by agencies. Only 75 patients give a history of using mosquito control measures with $25.7 \%$ using measures like mosquito coils and aerosols. None of them were using the biological measures or window meshes as preventive measures. $20.6 \%$ patients considered using long sleeved dresses as an effective preventive measures for preventing mosquito bites. Only $3 \%$ were using mosquito nets prior to infection but all of them used mosquito nets after the infection.

Table 3 Mosquito control measures used by the study population

\begin{tabular}{|l|c|c|}
\hline Mosquito control measures & Frequency & Percent \\
\hline Mosquito coil & 24 & 13.7 \\
\hline Liquids & 7 & 4 \\
\hline Aerosols & 14 & 8 \\
\hline Mosquito bats & 7 & 4 \\
\hline bed net & 3 & 1.7 \\
\hline Windows & 0 & 0 \\
\hline Repellents & 1 & 0.6 \\
\hline Long sleeved dress & 36 & 20.6 \\
\hline Herbal measure & 19 & 10.9 \\
\hline Biological measures & 0 & 0 \\
\hline
\end{tabular}


Cleaning premises

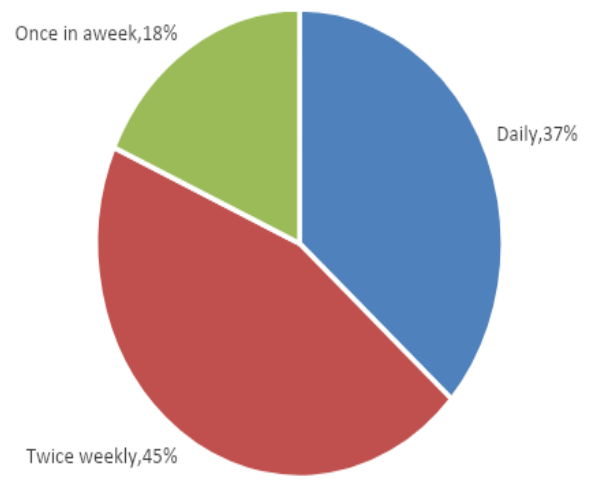

Fig 4 Cleaning practices among the study subjects

Table 4 Waste disposal measures used by the study population

\begin{tabular}{|l|c|c|}
\hline Methods of waste disposal & Frequency & Percent \\
\hline Composting & 7 & 4 \\
\hline Open dumping & 72 & 41.1 \\
\hline Collection by other agencies & 20 & 11.4 \\
\hline Burning & 112 & 64 \\
\hline
\end{tabular}

METHODS OF WASTE DISPOSAL

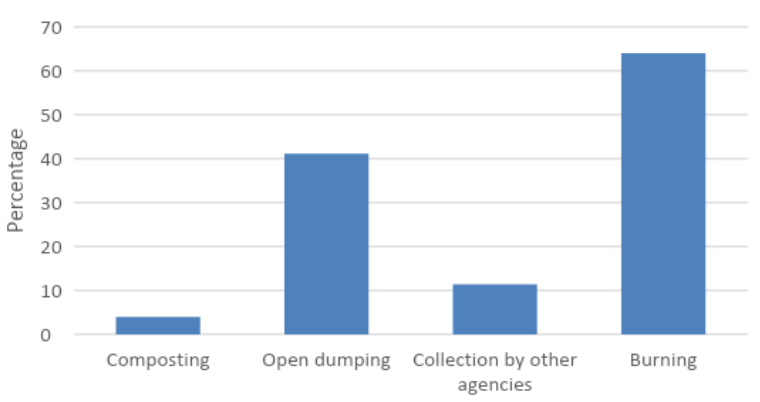

Fig 5 Waste disposal measures used by the study population

Majority of the cases did not have a previous history of dengue infection and the family history of dengue infection was elicited only in 40 $(22.3 \%)$ patients.

Table 5 Family history of dengue fever in the study population

\begin{tabular}{|l|c|c|}
\hline Family history & Frequency & Percent \\
\hline Yes & 40 & 22.9 \\
\hline No & 135 & 77.1 \\
\hline Total & 175 & 100 \\
\hline
\end{tabular}

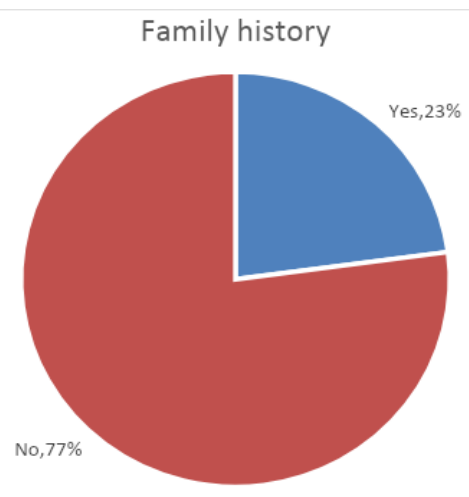

Fig 6 Family history of dengue fever in the study population

\section{Clinical features}

Fever was the most common presenting feature with $93.7 \%$ patient giving a history of high grade fever. Most of the patients were admitted between the $4^{\text {th }}$ and $7^{\text {th }}$ day of fever probably coinciding with onset of thrombocytopenia.

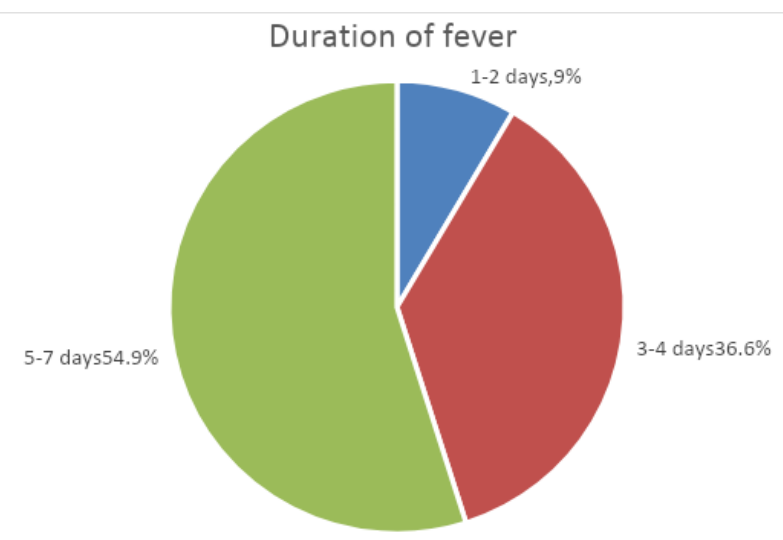

Fig 7 Duration of fever at the time of admission

Majority of the patients (54.9) were admitted 5-7 days after the onset of fever which usually corresponded to the development of thrombocytopenia which was also the most common indication for admission.

Table 6 Frequency of different symptoms among the study population

\begin{tabular}{|l|c|c|}
\hline Symptoms & Frequency & Percent \\
\hline Myalgia & 169 & 96.6 \\
\hline Retro orbital pain & 61 & 34.9 \\
\hline Headache & 132 & 75.4 \\
\hline Arthralgia & 44 & 25.1 \\
\hline Sore throat & 43 & 24.6 \\
\hline Rhinorrhoea & 54 & 30.9 \\
\hline Cough & 90 & 51.4 \\
\hline
\end{tabular}




\begin{tabular}{|l|c|c|}
\hline Breathing difficulty & 19 & 10.9 \\
\hline Calf pain & 11 & 6.3 \\
\hline Low back ache & 151 & 86.3 \\
\hline Nausea & 153 & 87.4 \\
\hline Abdominal pain & 75 & 42.9 \\
\hline Loose stools & 55 & 31.4 \\
\hline Jaundice & 1 & 0.6 \\
\hline Rashes & 28 & 16 \\
\hline Pruritus & 68 & 38.9 \\
\hline Clouding & 12 & 6.9 \\
\hline Conjunctival congestion & 48 & 27.4 \\
\hline Oliguria & 1 & 0.6 \\
\hline Seizures & 2 & 1.1 \\
\hline
\end{tabular}

Bleeding manifestations

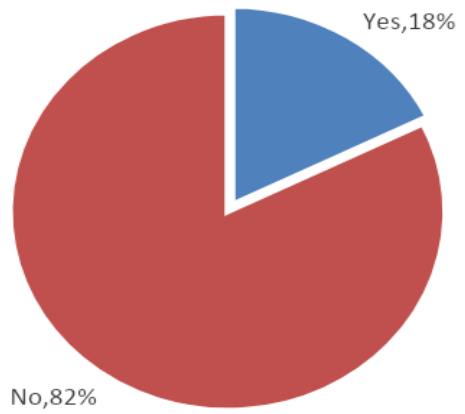

Fig 8 Frequency of bleeding manifestations in study population

Table 7 Bleeding manifestations seen among the study group

\begin{tabular}{|l|c|c|}
\hline Bleeding manifestations & Frequency & Percent \\
\hline Oral/ gum bleeding & 9 & 29.0 \\
\hline Skin bleed & 9 & 29.0 \\
\hline Hemarthroses & & 0.0 \\
\hline Epistaxis & 3 & 9.7 \\
\hline Hematuria & 7 & 22.6 \\
\hline Melena & 14 & 45.2 \\
\hline Hematemesis & 1 & 3.2 \\
\hline Menorrhagia & 10 & 32.3 \\
\hline
\end{tabular}

Myalgia (96.6\%) and low back ache (86.3\%) were very common symptoms. Majority of patients head ache $(75.4 \%)$ though the classically described retro orbital pain was present only in $34.9 \%$ of the study population. Arthralgia was seen in $25.1 \%$ of the patients. Upper respiratory symptoms were common with rhinorrhoea seen in $30.9 \%$ and sore throat in $24.6 \% .51 .4 \%$ of patient had cough but dyspnoea was present only in $10.9 \%$.

Among the gastrointestinal symptoms nausea was the most common symptom seen in $87.4 \%$ and loose stools were present in $31.4 \%$ patients Jaundice was an extremely rare symptom. Abdominal pain was a significant symptom seen in $75(42.9 \%)$ patients.

Rashes, which were maculopapular were seen only in $16 \%$ patients but pruritis was a common symptom seen in $38.9 \%$ patients. $17.7 \%$ of patients showed bleeding manifestations with melena (29\%) and superficial skin bleeding and oral bleeding as the most common bleeding sites. Among females menorrhagia was the most common bleeding manifestation.

Table 8 Examination findings in the study population

\begin{tabular}{|l|c|c|}
\hline General examination & Frequency & Percent \\
\hline Warmth of the extremities & 170 & 97.1 \\
\hline Pallor & 7 & 4 \\
\hline Conjunctival congestion & 63 & 36 \\
\hline Icterus & 2 & 1.1 \\
\hline Lymphadenopathy & 41 & 23.4 \\
\hline Odema & 50 & 28.6 \\
\hline JVP - Elevated & 20 & 11.4 \\
\hline
\end{tabular}

Lymphadenopathy, conjunctival congestion and edema were the most common signs. Bradycardia was seen in 24(13.7\%) patients. 18 (10.3\%) patients had features of lower respiratory tract infection. Epigastric tenderness was seen in $25(14.3 \%)$ patients. Hepatomegaly was clinically detected in 7 (4\%) patients. 2 patients had seizures but they were patients with seizure disorder that drug default and had no features of CNS involvement.

\section{Laboratory Parameters}

Table 9 Laboratory parameters among the study population

\begin{tabular}{|l|c|c|c|}
\hline & & Frequency & Percentage \\
\hline Hemoglobin & $\leq 11 \mathrm{~g} \%$ & 45 & 25.7 \\
\hline PCV & $>45 \%$ & 32 & 18.3 \\
\hline Total Count & $<4000$ & 60 & 34.3 \\
\hline & $4000-10000$ & 99 & 56.6 \\
\hline & $>10000$ & 10 & 5.7 \\
\hline Platelet & $<20000$ & 16 & 9.1 \\
\hline & $20000-50000$ & 71 & 40.6 \\
\hline & $50000-100000$ & 66 & 37.7 \\
\hline & $100001-150000$ & 12 & 6.9 \\
\hline & $>150000$ & 6 & 3.4 \\
\hline ESR & Elevated $>22$ & 43 & 24.6 \\
\hline & Normal & 127 & 72.6 \\
\hline
\end{tabular}




\begin{tabular}{|l|c|c|c|} 
Blood Urea & $<=20$ & 103 & 58.9 \\
\hline & $21-40$ & 59 & 33.7 \\
\hline & $41+$ & 4 & 2.3 \\
\hline & $>1.2$ & 16 & 9.1 \\
\hline sodium & $<135$ & 91 & 52.0 \\
\hline & $136-145$ & 73 & 41.7 \\
\hline & $>145$ & 1 & 0.6 \\
\hline Potassium & $<3.5$ & 19 & 10.9 \\
\hline & $3.5-5.0$ & 146 & 83.4 \\
\hline Bilirubin & $>5.0$ & 1 & 0.6 \\
\hline SGOT & $>2$ & 28 & 16.0 \\
\hline PT & $>40 \mathrm{IU} / \mathrm{L}$ & 147 & 84.0 \\
\hline ALP & $>40 \mathrm{IU} / \mathrm{L}$ & 115 & 65.7 \\
\hline Albumin & $<=3.5$ & 11 & 6.3 \\
\hline amylase & $>140$ & 103 & 58.9 \\
\hline & & 5 & 2.9 \\
\hline
\end{tabular}

\section{Haematological Parameters}

Anemia was seen in 45 (25.6\%) patients which of $>45 \%$ was seen in $32(18.3 \%)$ patients. $60(34.3 \%$ ) had leucopenia while only $5.7 \%$ had WBC count $>10,000 / \mathrm{cmm}$.ESR was normal in majority of patients.

153 patients had platelet count less than $1,00,000 / \mathrm{cmm}$. 71(40.6\%) patients had platelet count between $50,000 / \mathrm{cmm}$ and $20,000 / \mathrm{cmm}$. $16(19.3 \%)$ patients had platelet less than $20,000 / \mathrm{cmm}$. Bleeding manifestation and platelet count did not correlate as only 4 patients with platelet less than $20,000 / \mathrm{cmm}$ had bleeding. Bleeding manifestations were maximum when the platelet count was between $20,000 / \mathrm{cmm}$ and $50,000 / \mathrm{cmm} .17$ patients had bleeding manifestation when their platelet was in this range.

was present at the time of admission. Hematocrit

Table 10: Relation between platelet count and bleeding manifestation

\begin{tabular}{|c|c|c|c|c|c|c|c|c|c|}
\hline \multirow{3}{*}{ Platelet count } & \multicolumn{4}{|c|}{ Bleeding Manifestation } & \multirow{2}{*}{\multicolumn{2}{|c|}{ Total }} & \multirow{3}{*}{$\chi^{2}$} & \multirow{3}{*}{$\mathrm{df}$} & \multirow{3}{*}{$\mathrm{p}$} \\
\hline & \multicolumn{2}{|c|}{ Yes } & \multicolumn{2}{|c|}{ No } & & & & & \\
\hline & $\mathrm{N}$ & $\%$ & $\mathrm{~N}$ & $\%$ & $\mathrm{~N}$ & $\%$ & & & \\
\hline$<20000$ & 4 & 25.0 & 12 & 75.0 & 16 & 100.0 & \multirow{5}{*}{5.947} & \multirow{5}{*}{4} & \multirow{5}{*}{.203} \\
\hline $20000-50000$ & 17 & 23.9 & 54 & 76.1 & 71 & 100.0 & & & \\
\hline $50000-100000$ & 8 & 12.1 & 58 & 87.9 & 66 & 100.0 & & & \\
\hline $100001-150000$ & 1 & 8.3 & 11 & 91.7 & 12 & 100.0 & & & \\
\hline$>150000$ & 0 & 0.0 & 6 & 100.0 & 6 & 100.0 & & & \\
\hline Total & 30 & 17.5 & 141 & 82.5 & 171 & 100.0 & & & \\
\hline
\end{tabular}

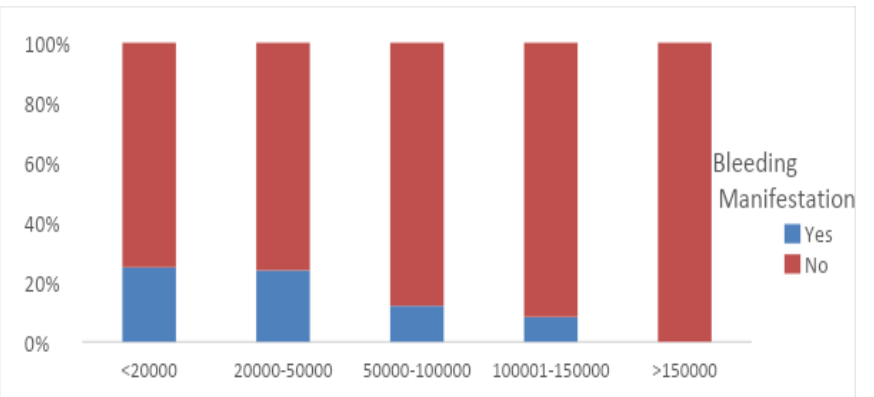

Fig 9: Relation between platelet count and bleeding manifestation

\section{Biochemical parameters}

Renal function tests were normal in majority of patients.

Bilirubin $>2 \mathrm{mg} \%$ was seen in 28 patients but only 2 of them had value $>5 \mathrm{mg} \%$. The major abnormality in LFT was a raised liver transaminases. AST (SGOT) >40IU/l were seen in $147(84 \%)$ patients and elevated ALT (SGPT) in
115(65.7\%) patients. ALP >126IU/L was seen only in $11(6.3 \%)$ patients.

Amylase was done in 18 patients with abdominal pain but was only mildly elevated in 5 patients which were not significant. S. lipase was normal in these patients.

\section{Other Investigations}

Dengue NS1 Ag test was positive in 115 patients (65.7\%) and IgM Dengue antibody test by ELISA was positive in 60 patients(34.3\%).

Chest X-ray was done in 10 patients, 3 patients had mild bilateral pleural effusion and 2 had pneumonia.

USG abdomen was done in 12 patients, out of which 2 patients had acalculous cholecystitis., 8 (4.5\%) had ascites and 9(5.1\%) had hepatomegaly. 5 patients had pleural effusion 
detected sonologically. 5 patients had polyserositis.

\section{Duration of Hospital Stay}

Average duration of hospital stay was $5.36 \pm 1.36$ days an ranging from 2 to 12 days

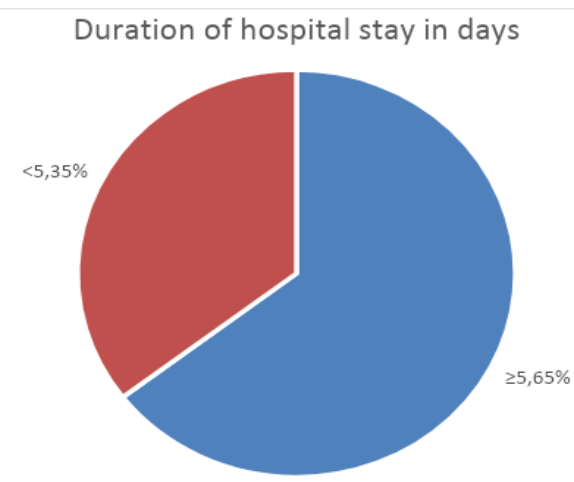

Fig 10 Duration of Hospital Stay

\section{Transfusion Trends}

$21(12 \%)$ patients had platelet transfusion. The most common indication was bleeding manifestation .5 patients had transfusion when platelet count was less than $15,000 / \mathrm{cmm}$ even without bleeding.

On Follow up of 154 patients who turned up for review in OPD at first week and $2^{\text {nd }}$ week the major complaint was fatigue followed by persistent myalgia .Insomnia was an important symptom especially in the middle aged .

Table 11: Follow up evaluation in patients

\begin{tabular}{|l|c|c|}
\hline Symptoms on follow up & Frequency & Percent \\
\hline Fatigue & 144 & 88 \\
\hline Myalgia & 90 & 51.4 \\
\hline Arthralgia & 22 & 12.6 \\
\hline Insomnia & 40 & 22.9 \\
\hline
\end{tabular}

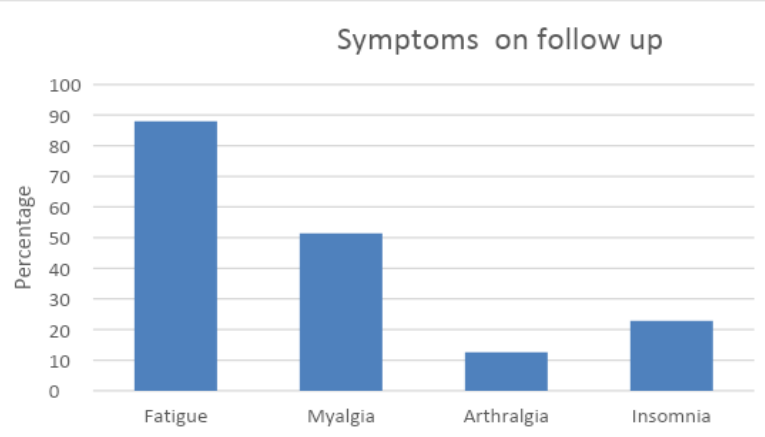

Fig 11: Follow up evaluation in patients

\section{Outcome in the Study Populations}

There was no mortality reported in the present study probably due to admission bias from the emergency room as patients with multi organ dysfunction like renal or hepatic failure were referred to the nearest $\mathrm{MCH}$ with super speciality facilities like dialysis .This could also reflect the increased awareness among health care workers and the availability of rapid diagnostic tests that can be positive from the $2^{\text {nd }}$ day of fever itself resulting in prompt diagnosis and effective management.

\section{Limitation of the Study}

Single centre study

No mortality was reported probably hence mortality predictors of dengue could not be analysed.

\section{Discussion}

This study describes the clinical profile, laboratory features and outcome of DF in adult patients admitted in GMC kollam.83.3\% were classified as dengue fever and $18.7 \%$ were DHF/DSS. Majority of DF cases were in the productive age group between $21-50$ years underlying the public health and financial impact of dengue. Men were more frequently affected than women but among the $30-50$ year age group females were more commonly infected. A significant proportion of the females affected were homemakers underlying the fact that domestic premises were the most common source for breeding of A.aegypti.

Majority of the patients had disabling myalgia, low backache and headache. Deshwal et al in their study noted myalgia and headache in more than 90/ of their study population ${ }^{[8]}$. Low backache independent of myalgia was a significant symptom seen in $80.6 \%$ patients which was not mentioned in any of the previous studies in India. Retro-orbital pain as a cardinal feature of dengue fever was seen in few (34.9\%) of our patients.

A significant finding was the increased prevalence of upper respiratory symptoms like sore throat 
(24.6\%), rhinorrhoea (30.4\%) and cough (51.4\%) in the study population. In a similar study by Rachel Daniel et al. Sore throat was seen only in $5.2 \%$ of patients ${ }^{[6]}$. Ramesan $\mathrm{K}$ et al. studied the profile of dengue cases in north Kerala and reported the prevalence of cough as only $15 \%{ }^{[5]}$. In a study from Nimmannitya et al. around $96 \%$ of patients had congested pharynx, and rhinitis was reported in $13 \%$ of the patients ${ }^{[10]}$. These symptoms were predominantly seen in the first few days of fever and thus symptoms can mimic influenza at their initial presentation in the present epidemic.

Gastrointestinal symptoms were also significantly higher compared to previous studies. Diarrhoea (31.4\%) and abdominal pain (42.9\%) were two important GI manifestations. All the previous studies in India (Ramesan et al, Varsha et al, Rajesh et al) reported the incidence of diarrhoea as less than $20 \%$. Thus other infections that cause fever and gastrointestinal symptoms such as typhoid, and enteroviral infections that are common in India may often lead to a delay in the diagnosis of dengue.

Another significant finding was pruritis seen in $38.9 \%$ of patients even in the absence of rashes and features of cholestasis. Presence of rashes $(16 \%)$ was lower in the present study compared to studies from other parts of the country but comparable to the study by Rachel et al in the same geographical area (33 patients) ${ }^{[6]}$.

Thus a combination of fever severe myalgia, low backache with diarrhoea and upper respiratory symptoms should raise the possibility of dengue in a patient.

Bleeding manifestations were significantly lower compared to the previous studies and the most common were bleeding from GI sites. An in increase in menstrual bleeding was a significant finding in women. Thrombocytopenia was a common finding as it was the common indication for admission in our centre. However there was poor correlation between thrombocytopenia and bleeding tendencies, an observation similar to the one made by Sharma et al ${ }^{[11]}$ and Rachel et al. The study by Rajesh D et al, from Agra showed 14.8\% patients had hepatomegaly which was significantly higher in patients with DHF and DSS. Generally, it was reported that more than $90 \%$ of Asian subjects with DHF have hepatomegaly. Thus the lower incidence of hepatomegaly could be attribute to the less number of DHF cases. Liver function abnormalities, especially elevated transaminases, were noted in this study. $84 \%$ had SGOT $>40 \mathrm{IU} / \mathrm{L}$. Three fold elevation of SGOT was seen in $40.5 \%$ patients. Dengue virus-induced damage to the hepatocytes, hypoxia, shock or associated liver disease have all been postulated to be the pathogenic mechanisms for the occurrence of transaminitis. No case of fulminant hepatic failure was noted in our study though 2 patients had SGOT >1000 IU/L. The series from Sharma et al. from India reported elevated transaminases in $90 \%$ of patients ${ }^{[11]}$. In study by Kularatne et al, $88 \%$ patients showed elevated ALT and AST, with 122 of them having a two fold increase ${ }^{[12]}$. Mandal et al documented elevated transaminases in $83.78 \%$ of cases $^{[7]}$. Liver enzyme elevation, a common feature in dengue infection was also apparent in a study done by Mohan et $\mathrm{al}^{[9]}$. No mortality were reported in our study similar to the study by. Md. Yousuf Khan et al which indicate prompt diagnosis by health community due to availability of early antigen detection tests and increased awareness about dengue ${ }^{[13]}$.

An important aspect of the study was to assess the awareness of mosquito control measures among the study populations. Only 4\%of the study population practised dry day at homes and open dumping of wastes were practised by $41 \%$ of the study population. Mosquito net use was prevalent only in $1.7 \%$ but A.aegypti being predominantly day time biter its impact on dengue spread cannot be ascertained.

\section{Conclusion}

This study has revealed a varied clinical profile of dengue fever which is of important diagnostic value. In the recent few years, the world has seen 
varied clinical presentation of the dengue fever in different epidemics, in the same regions. Some of the classical findings are still manifesting but their frequency is variable between epidemics. Few atypical features are also reported from different parts of the world like the higher incidence of upper respiratory symptoms and diarrhoea in the present study. Continuous seroepidemiological surveillance and clinical profile studies are needed for the early detection of cases with atypical features. This study also stresses on the lack of awareness for the mosquito control measures among public and need for more interventions in the vector control.

\section{References}

1. Special Programme for Research. Dengue: Guidelines for Diagnosis, Treatment, Prevention and Control. Geneva, Switzerland: World Health Organization; 2009.

2. WHO. Factsheet no. 117 . Geneva, Switzerland: World Health Organization; 2008. Dengue and dengue haemorrhagic fever. yyu

3. Gupta N., Srivastava S., Jain A., Chaturvedi U. C. Dengue in India. Indian Journal of Medical Research. 2012; 136(3):373-390. [PMC free article] [PubMed]

4. CDC. Imported dengue- United States, 1997 and 1998. Morb Mortal Wkly Rep. 2000;49:248-5.

5. Ramesan K, Kumar MKA, Valliyot B, Balakrishnan S. Clinical profile of dengue fever in a tertiary care centre in North Kerala. Int J Res Med Sci 2017;5:4297301.

6. Rachel D, Rajamohanan, Philip AZ. A study of clinical profile of dengue fever in Kollam, Kerala, India. Dengue Bulletin. 2005;29:197-202.
7. Mandal SK, Ganguly J, Koelina Sil. Clinical profiles of dengue fever in a teaching hospital of eastern India. Nat $\mathbf{J}$ Med Res. 2013;3:173-6.

8. Deshwal R, Qureshi MI, Singh R. Clinical and laboratory profile of dengue fever. J Associat Physic India.2015;63:30-2.

9. Mohan DK, Shiddappa, Dhananjaya M. A study of clinical profile of dengue fever in a tertiary care teaching Hospital. Sch J AppMed Sci. 2013;1:280-2.

10. Nimmanitya $S$ and Kalayanarooj $S$. Guidelines for DHF case management for workshop on case management of DHF, Queen Sirikit National Institute For Child Health, Ministry of Public Health, Bangkok, Thailand, 2002.

11. Sharma S and Sharma SK. Clinical profile of DHF in adults during 1996 outbreak in Delhi, India. Dengue Bulletin. 1998; 22: 20-

12. Kularatne SA, Gawarammana IB, Kumarasiri PR. Epidemiology, clinical features, laboratory investigations and early diagnosis of dengue fever in adults: a descriptive study in Sri Lanka. Southeast Asian J Trop Med Public Health 2005; 36:686- 92.

13. Md. Yousuf Khan, C.Venkateshwarlu, N. Sandeep, A Hari Krishna. A study of clinical and laboratory profile of dengue fever in a tertiary care hospital, Nizamabad, Telangana State, India. International Journal of Contemporary Medical Research 2016;3(8):2383-2387.

14. Kelly JD, Shandera WX. Viral and Rickettsial Infections. In: Papadakis MA, McPhee SJ, Rabow MW, editors. 2016: Current Medical Diagnosis and Treatment. 55th ed. McGraw-Hill Education; New York: 2016.pp. 1342-416. 